\title{
IRREDUCIBLE OUTER AUTOMORPHISMS OF A FREE GROUP
}

\author{
S. M. GERSTEN AND J. R. STALLINGS
}

(Communicated by Frederick R. Cohen)

\begin{abstract}
We give sufficient conditions for all positive powers of an outer automorphism of a finitely generated free group to be irreducible, in the sense of Bestvina and Handel. We prove a conjecture of Stallings (1982), that a $P V$ automorphism in rank $\geq 3$ has no nontrivial fixed points.
\end{abstract}

\section{INTRODUCTION}

A map of finite graphs $f: G \rightarrow G$ will be required to take vertices to vertices and map each edge to a path in $G$ (thus $f^{-1}(V)$ is not required to be $V$, where $V$ is the vertex set of $G$ ). If $G$ is connected, and if $f$ is a homotopy equivalence with $f \mid E$ an immersion for each edge $E$ of $G$, then $f$ is said to be a topological realization of the outer automorphism $\mathscr{O}(f)$ of $\pi_{1}(G, x)$ (for $x \in V)$ determined by $\alpha \mapsto p \circ f(\alpha) \circ p^{-1}$; here $p$ is a path from $x$ to $f(x)$ and $\alpha$ is a loop based at $x$. It is readily checked that $\mathscr{O}(f)$ is independent of the choices of $x$ and $p$.

An outer automorphism $\mathscr{O}$ of a finitely generated free group is said to be reducible (in the sense of Bestvina-Handel [BH]) if $\mathscr{O}=\mathscr{O}(f)$ where $f: G \rightarrow G$ is a topological realization such that (a) $G$ has no vertices of valence one, (b) no $f$-invariant forest of $G$ contains an edge, and (c) there exists an $f$-invariant subgraph $G_{0}$ of $G$ (with $G_{0}$ not necessarily connected) so that $G_{0} \neq G$ and so that $\pi_{1}\left(G_{0}, x\right) \neq\{1\}$ for some vertex $x$ of $G_{0}$. If $\mathscr{O}$ is not reducible it is called irreducible. It is an easy matter to check whether $\mathscr{O}$ is reducible; one need only produce one graph $G$ as in the definition. However it is by no means an easy matter to check irreducibility. We shall describe some classes of irreducible automorphisms and apply thereto a beautiful result [BH, Theorem 4.1]:

Theorem. Suppose $\mathscr{O}$ is an outer automorphism of a finitely generated free group $F$ such that every power $\mathscr{O}^{k}, k \geq 1$, is irreducible. If there is a nontrivial cyclic word $s \in F$ such that either $\mathscr{O}(s)=s$ or $\mathscr{O}(s)=s^{-1}$, then $\mathscr{O}$ is geometric.

Received by the editors June 2, 1989.

1980 Mathematics Subject Classification (1985 Revision). Primary 20F28; Secondary 57M05.

This paper is based on research partly supported by the National Science Foundation under grants No. DMS-860-1376 (Gersten), DMS-860-0320 (Stallings), and DMS-850-5550 (Mathematical Sciences Research Institute). 
That $\mathscr{O}$ is geometric means that it is the homomorphism induced on fundamental group by an autohomeomorphism of a surface. In the conclusion of the above theorem, the surface involved has exactly one boundary component. (The statement in $[\mathrm{BH}]$ carries the additional hypothesis that the FrobeniusPeron eigenvalue $\lambda$ associated to a train track topological representative for $\mathscr{O}$ should satisfy $\lambda>1$; however if $\lambda=1$, the train track map is of finite order, and so if the rank of $F$ is greater than one, $\mathscr{O}^{k}$ must be reducible for some $k \geq 1$.)

An outer automorphism $\mathscr{O}$ of a free group $F_{n}$ of rank $n$ determines an automorphism $\mathscr{O}_{a b}$ of the abelianization $\left(F_{n}\right)_{a b} \cong \mathbf{Z}^{n}$; if we choose a basis for $\mathbf{Z}^{n}$ we obtain a matrix representation for $\mathscr{O}_{a b}$. Let char $\mathscr{O}$ denote the characteristic polynomial of $\mathscr{O}_{a b}$.

Recall that $p(x) \in \mathbf{Z}[x]$ is called a $P V$-polynomial if it is monic and if has precisely one root $\lambda$ (counted with multiplicity) with $|\hat{\lambda}|>1$, whereas for all other roots $\mu,|\mu|<1$. (The large root $\lambda$ is a Pisot-Vijayaraghavan number.)

In what follows, a polynomial is said to be irreducible if it is irreducible over the rationals. We shall show:

Corollary 2.6. If char $\mathscr{O}$ is a PV-polynomial, then $\mathscr{O}^{k}, k \geq 1$, is irreducible.

If $M$ is a square matrix of real numbers, say $M$ is primitive if each entry of $M$ is nonnegative and if $\exists N \geq 1$ such that every entry of $M^{N}$ is positive.

Corollary 2.7. If char $\mathscr{O}$ is irreducible and if some matrix representation for $\mathscr{O}_{a b}$ is primitive, then, for all $k \geq 1, \mathscr{O}^{k}$ is irreducible.

Given an automorphism $\phi: F \rightarrow F$, we define the fixed subgroup, $\operatorname{Fix}(\phi)=$ $\{w \in F \mid \phi(w)=w\}$. Finally, we settle a conjecture of [St]:

Corollary 2.8. If $\phi$ is an automorphism of a finitely generated free group of rank $\geq 3$, such that char $\phi$ is a PV-polynomial, then $\operatorname{Fix}(\phi)=\{1\}$.

\section{THE DETAILS}

Let $p(x) \in \mathbf{Q}[x]$ be a monic polynomial of degree $n$ with rational coeffcients, and with complex roots $\lambda_{1}, \ldots, \lambda_{n}$. Thus, $p(x)=\prod_{1}^{n}\left(x-\lambda_{i}\right)$. We say that $p(x)$ is super irreducible, when, for every integer $k \geq 1$, the polynomial $p_{k}(x)=\prod_{1}^{n}\left(x-\lambda_{i}^{k}\right)$ is irreducible in $\mathbf{Q}[x]$. We say that an $n \times n$ matrix $M$ over $\mathbf{Q}$ is super irreducible, when its characteristic polynomial char $M$ is super irreducible; equivalently, as a linear transformation $M: \mathbf{Q}^{n} \rightarrow \mathbf{Q}^{n}$, no positive power $M^{k}$ maps a proper subspace of $\mathbf{Q}^{n}$ into itself.

2.1. Theorem. A monic irreducible polynomial $p(x) \in \mathbf{Q}[x]$ with roots $\lambda_{1}, \ldots$, $\lambda_{n}$ is super irreducible if and only if, for all $i, j$ with $1 \leq i<j \leq n$, the quotient $\lambda_{i} \cdot \lambda_{j}^{-1}$ is not a root of unity. 
Proof. Let $\Gamma$ denote the Galois group of the polynomial $p(x)$ over $\mathbf{Q}$. Since $p(x)$ is irreducible, $\Gamma$ acts transitively on the roots $\left\{\lambda_{i}\right\}$. Therefore $\Gamma$ acts transitively on the $k$ th powers of these roots, which are the roots of $p_{k}(x)$. Thus, $p_{k}(x)$ is irreducible, if and only if, whenever $i \neq j$, then $\lambda_{i}^{k} \neq \lambda_{j}^{k}$; and this occurs if and only if $\lambda_{i} \cdot \lambda_{j}^{-1}$ is not a $k$ th root of unity.

2.2. Theorem. If the monic polynomial $p(x) \in \mathbf{Q}[x]$ with roots $\left\{\lambda_{i}\right\}$ is irreducible, and if, for all $i \neq 1,\left|\lambda_{1}\right|>\left|\lambda_{i}\right|$, then $p(x)$ is super irreducible.

Proof. As before, the Galois group $\Gamma$ acts transitively on the roots, so that for any $i \neq j$, there is $\sigma \in \Gamma$ such that $\sigma\left(\lambda_{i}\right)=\lambda_{1}$, and $\sigma\left(\lambda_{j}\right)=\lambda_{r}$ for $r \neq 1$. Then

$$
\left|\sigma\left(\lambda_{i} \cdot \lambda_{j}^{-1}\right)\right|=\left|\lambda_{1} \cdot \lambda_{r}^{-1}\right|>1,
$$

so that $\sigma\left(\lambda_{i} \cdot \lambda_{j}^{-1}\right)$ cannot be a root of unity, and therefore $\lambda_{i} \cdot \lambda_{j}^{-1}$ cannot be a root of unity. Thus Theorem 2.1 applies.

2.3. Corollary. If $p(x)$ is a PV-polynomial, then $p(x)$ is super irreducible.

Proof. $p(x)$ is a monic integer polynomial having a unique root of absolute value greater than 1 , the other roots having absolute value less than 1 . Since any monic integer polynomial has at least one root of absolute value at least 1 , the polynomial $p(x)$ must be irreducible. Theorem 2.2 immediately applies to show that $p(x)$ is super irreducible.

2.4. Corollary. If $M$ is a primitive matrix of rational numbers with char $M$ irreducible, then char $M$ is super irreducible.

Proof. From Perron's theorem [Ga, p. 53] one knows that $M$ possesses a positive real eigenvalue $\lambda$ of multiplicity one such that all other eigenvalues $\mu$ of $M$ satisfy $|\mu|<\lambda$. With the assumption that $\operatorname{char} M$ is irreducible, Theorem 2.2 then applies to show that $\operatorname{char} M$ is super irreducible.

Recall that if $\mathscr{O}$ is an outer automorphism of a finitely generated free group $F$, then $\operatorname{char} \mathscr{O}$ is the characteristic polynomial of the abelianization of $\mathscr{O}$.

2.5. Theorem. If char $\mathscr{O}$ is super irreducible, then for every integer $k \geq 1$, the power $\mathscr{O}^{k}$ is an irreducible outer automorphism (in the sense of Bestvina and Handel).

Proof. By assumption, char $\mathscr{O}^{k}$ is irreducible for all $k \geq 1$. Suppose that $\mathscr{O}$ were reducible in the sense of Bestvina and Handel. Then there exists a topological representative $f: G \rightarrow G$ for $\mathscr{O}=\mathscr{O}(f)$, with $G$ a connected graph having no vertex of valence 1 , having only subsets of vertices as $f$-invariant forests, and possessing a subgraph $G_{0} \neq G$ with $f\left(G_{0}\right) \subseteq G_{0}$ and $\pi_{1}\left(G_{0}, x\right) \neq$ \{1\} for some vertex $x$. If $G_{0}^{\prime}$ is the connected component of $G_{0}$ containing $x$, there exist $l, k>0$ such that $f^{k+l}\left(G_{0}^{\prime}\right) \subseteq f^{l}\left(G_{0}^{\prime}\right)$; in fact, we can choose $k$ and $l$ so that $f^{k}$ fixes a vertex $f^{l}(x)$ of $G_{0}$. Then $\pi_{1}\left(f^{l}\left(G_{0}^{\prime}\right), f^{l}(x)\right)$ is 
a proper free factor of $\pi_{1}\left(G, f^{l}(x)\right)$ which is invariant under $f_{*}^{k}$. It follows that the matrix for $\left(f_{*}^{k}\right)_{a b}$ can be written in block form

$$
\left(\begin{array}{ll}
* & * \\
0 & *
\end{array}\right) \text {. }
$$

In particular char $\mathscr{G}^{k}$ would be reducible; this contradicts the assumption that char $\mathscr{O}$ is super irreducible. We can apply the same argument to $\mathscr{O}^{r}$ for any $r \geq 1$ to show that $\mathscr{O}^{r}$ is irreducible.

2.6. Corollary. If char $\mathscr{O}$ is a $P V$-polynomial, then $\mathscr{O}^{k}$, for all $k \geq 1$, is irreducible.

Proof. This follows from Corollary 2.3 and Theorem 2.5.

2.7. Corollary. If char $\mathscr{O}$ is irreducible and if some matrix representation for $\mathscr{O}_{a b}$ is primitive, then, for all $k \geq 1, \mathscr{\sigma}^{k}$ is irreducible.

Proof. Corollary 2.4 plus Theorem 2.5.

2.8. Corollary. If $\phi$ is an automorphism of a finitely generated free group $F_{n}$ of rank $n \geq 3$, such that char $\phi$ is a $P V$-polynomial, then $\operatorname{Fix}(\phi)=\{1\}$.

Proof. By Corollary 2.6, if $\mathscr{O}$ is the outer automorphism represented by $\phi$, then all positive powers $\mathscr{O}^{k}$ are irreducible in the sense of Bestvina and Handel. If $\operatorname{Fix}(\phi)$ were nontrivial, there would exist $s \in F_{n}$ with $s \neq 1$ such that $\phi(s)=s$. By Theorem 4.1 of $[\mathrm{BH}]$, quoted in the introduction, $\mathscr{O}$ would be geometric, induced by a homeomorphism of a compact surface with one boundary component. However, in [St] it is proved that if the rank of $F_{n}$ is at least 3, then a $P V$ outer automorphism is not induced by a homeomorphism of any bounded compact surface, regardless of the number of components of the boundary. This contradiction shows that $\operatorname{Fix}(\phi)=\{1\}$.

2.9. Corollary. If $\phi$ is a PV-automorphism of a finitely generated free group of rank $\geq 3$, then $\phi$ has no nontrivial recurrent words.

Proof. The set of recurrent words is by definition $\bigcup_{k \geq 1} \operatorname{Fix}\left(\phi^{k}\right)$. But $\operatorname{Fix}\left(\phi^{k}\right)=$ $\{1\}$ since $\phi^{k}$ is $P V$.

\section{REMARKS}

3.1. Example. In Theorem 2.5 we need the hypothesis that for all $k \geq 1$ the polynomial char $\mathscr{\sigma}^{k}$ is irreducible. The hypothesis that char $\mathscr{\theta}$ alone is irreducible does not imply that $\mathscr{O}$ is irreducible, as the following example shows. Let $G$ be the graph with two vertices $P$ and $Q$, and three edges $A, B$, and $C$; suppose that $C$ joins $P$ to $Q$, that $A$ joins $P$ to $P$, and that $B$ joins $Q$ to $Q$; thus $G$ is a "pince-nez" graph, with two circles $A$ and $B$ and a midbar $C$. Let $f: G \rightarrow G$ be given by $P \mapsto Q, Q \mapsto P, A \mapsto B, B \mapsto \bar{A}, C \mapsto B \bar{C} A$. 
Observe that there are no vertices of valence one and that $\{P, Q\}$ is the only $f$-invariant forest. However $G_{0}=\{A, B, P, Q\}$ is an $f$-invariant subgraph. Thus $\mathscr{O}(f)$ is reducible. We can calculate char $\mathscr{O}(f)$ with respect to the homology basis $\alpha=[A], \beta=[B]$ to get $\operatorname{char} \mathscr{O}(f)=x^{2}+1$, which is irreducible.

3.2. Example. The characteristic polynomial for the matrix $M$ given by

$$
M=\left(\begin{array}{ccccc}
0 & 1 & 0 & \cdots & 0 \\
0 & 0 & 1 & \cdots & 0 \\
\vdots & & & \ddots & \\
0 & 0 & 0 & \cdots & 1 \\
1 & 1 & 1 & \cdots & 1
\end{array}\right)
$$

is $P V$, [Ge, p. 87]. It follows that if $\mathscr{O}$ is an outer automorphism such that $\mathscr{O}_{a b}$ has $M$ as a matrix representation, then $\mathscr{O}^{k}$ is irreducible for all $k \geq 1$.

3.3. Example. Consider the $4 \times 4$ integral matrix

$$
M=\left(\begin{array}{cccc}
0 & 1 & 0 & 0 \\
0 & 0 & 1 & 0 \\
0 & 0 & 0 & 1 \\
1 & b_{1} & b_{2} & b_{3}
\end{array}\right) .
$$

This matrix is primitive if all $b_{i}>0$. The characteristic polynomial is char $M=$ $x^{4}-b_{3} x^{3}-b_{2} x^{2}-b_{1} x-1$. We shall determine when char $M$ is irreducible and hence when Corollary 2.7 applies to an outer automorphism $\mathscr{\theta}$ with $\operatorname{char} \mathscr{\theta}=$ char $M$. The only possible linear factor of char $M$ is $x+1$ (since +1 is not a root) and this leads to the equation $b_{2}=b_{1}+b_{3}$. A factorization with quadratic factors is of the form

$$
\operatorname{char} M=\left(x^{2}+\alpha x+\beta\right)\left(x^{2}+\gamma x+\delta\right) .
$$

This leads to equations

$$
\begin{aligned}
\alpha+\gamma & =-b_{3}, \\
\alpha \gamma+\beta+\delta & =-b_{2}, \\
\alpha \delta+\beta \gamma & =-b_{1}, \\
\beta \delta & =-1 .
\end{aligned}
$$

Since $\beta+\delta=0$ these equations simplify and we get $b_{1}^{2}=b_{3}^{2}+4 b_{2}$. Thus char $M$ is irreducible iff both $b_{2} \neq b_{1}+b_{3}$ and $b_{1}^{2} \neq b_{3}^{2}+4 b_{2}$.

In the easier case of the matrix

$$
M_{1}=\left(\begin{array}{ccc}
0 & 1 & 0 \\
0 & 0 & 1 \\
1 & b_{1} & b_{2}
\end{array}\right)
$$

where $b_{1}$ and $b_{2}$ are positive integers, char $M_{1}$ is irreducible iff $b_{1} \neq b_{2}+2$. In this case, either $\operatorname{char} M_{1}$ or $\operatorname{char} M_{1}^{-1}$ is $P V$. 
3.4. Question. Finally we would like to raise a question about the split extension $E=F \times_{\mathscr{O}} \mathbf{Z}$, where $F$ is a finitely generated free group and $\mathscr{O}$ is an outer automorphism of $F$. If for some $h \geq 1, \mathscr{\sigma}^{k}$ fixed a nontrivial cyclic word, then $E$ would possess a free abelian subgroup of rank 2 and hence $E$ would not be word hyperbolic in the sense of Gromov [Gr]. A bold conjecture would be: If for all $k \geq 1, \mathscr{O}^{k}$ is irreducible and fixes no nontrivial cyclic word, then the split extension $E$ is word hyperbolic.

Perhaps one should first try to see if this is true when $\mathscr{O}$ is $P V$.

\section{REFERENCES}

[BH] M. Bestvina and M. Handel, Train tracks and automorphisms of free groups (to appear).

[Ga] F. R. Gantmacher, The theory of matrices, Vol. II, Chelsea, New York, 1959.

[Ge] S. M. Gersten, On fixed points of certain automorphisms of free groups, Proc. London Math. Soc. 48 (1984), 72-90.

[Gr] M. Gromov, Hyperbolic groups, Essays in Group Theory (S. M. Gersten, ed.), MSRI Publication, vol. 8, Springer-Verlag, 1987, pp. 75-263.

[St] J. R. Stallings, Topologically unrealizable automorphisms of free groups, Proc. Amer. Math. Soc. 84 (1982), 21-24.

Department of Mathematics, University of Utah, Salt Lake City, Utah 84112

Department of Mathematics, University of California, Berkeley, California 94720 